\title{
Sarcoglycan, the heart, and skeletal muscles: new treatment, old drug?
}

\section{Commentary}

See related article, pages $\mathrm{R} 1-\mathrm{R} 7$.

\author{
Jeffrey A. Towbin ${ }^{1,2}$ and $\mathrm{N}$ eil E. Bowles ${ }^{1}$
}

${ }^{1}$ D epartment of Pediatrics (Cardiology), and

2D epartment of Molecular and Human Genetics, Baylor College of M edicine, Houston, Texas, USA

Address correspondence to: J effrey A. Towbin, Pediatric Cardiology, Baylor College of M edicine, One Baylor Plaza,

Room 333E, H ouston, Texas 77030, USA. Phone: (713) 798-7342; Fax: (713) 798-8085; E-mail: jtowbin@bcm.tmc.edu.

In this issue of the $\mathrm{JCl}$, Cohn and colleagues describe important findings regarding the development of dilated cardiomyopathy (DCM ) and a potential therapeutic option that can alleviatethe cardiomyopathic phenotype (1). Using mouse models of cardiomyopathy in which they ablatetwo of the sarcoglycan complex members, $\beta$-sarcoglycan and $\delta$ sarcoglycan, and thereby disrupt the muscle cytoskeleton and the sarcoglycan-sarcospan complex in vascular smooth muscle(2), theauthors demonstrate improvement of the signs and symptoms using the calcium channel blocker/vasodilator verapamil (1). This improvement includes normalization of the myocardial morphology and serum cardiac troponin I levels. As proof of concept, the authors also show that interruption of verapamil therapy leads to vascular dysfunction, acute myocardial necrosis, and elevation of serum troponin I. Importantly, verapamil therapy in dystrophin-deficient mdx mice, which havean intact sarcoglycan complex, was unableto prevent cardiac musclepathology. These data suggest that verapamil therapy in patients with certain forms of cardiomyopathy, particularly those caused by sarcoglycan mutations or associated with secondary sarcoglycan deficiency, could be effective in alleviating the signs, symptoms, and devastating outcome of patients with cardiomyopathies. How does this animal model study impact human patients? Let us evaluatetheimportance of this work.

Over the past several years, multiple genes have been reported to causeDCM, including dystrophin (X-linked DCM) (3), actin (4), desmin (5), lamin A/C (6, $7)$, and, most recently, $\delta$-sarcoglycan (8). These genes not only appear to contribute to structural support of the myocyte, but al so appear to link the sarcomere to the sarcolemma and ECM. Dystrophin plays a major role by connecting to the sarcomere via the actin cytoskeleton at the $\mathrm{N}_{2}$-terminal end of dystrophin and to the sarcolemma at the $\mathrm{COOH}$-terminal through its interaction with the oligomeric membrane complex known as the dystrophin-associated protein complex (DAPC), which includes the sarcoglycan $(\alpha, \beta, \gamma, \delta, \varepsilon)$ and dystroglycan $(\alpha, \beta)$ subcomplexes (9) (Figure 1). Interestingly, all of the known DCM-causing genes have been previously shown to cause skeletal myopathy, and mutations in the DAPC proteins are important causes of skeletal muscledisease $(8,10)$. M ousemodels with defectivesarcoglycan genes haveall shown associated cardiomyopathy and skeletal myopathy (limb-girdle muscular dystrophy in mice and humans), while patients with sarcoglycan deficiency have associated DCM in about $30 \%$ of cases (11). In the $\delta$-sarcoglycan-deficient mouse, abnormalities of the coronary arteries were also found, resulting in ischemia (2). The mechanism has been speculated to be disruption of the sarcoglycan-sarcospan complex in vascular smooth muscle. In contradistinction, patients with heterozygous $\delta$-sarcoglycan mutations resulting in autosomal dominant $D C M$ or sporadic DCM had normal coronary

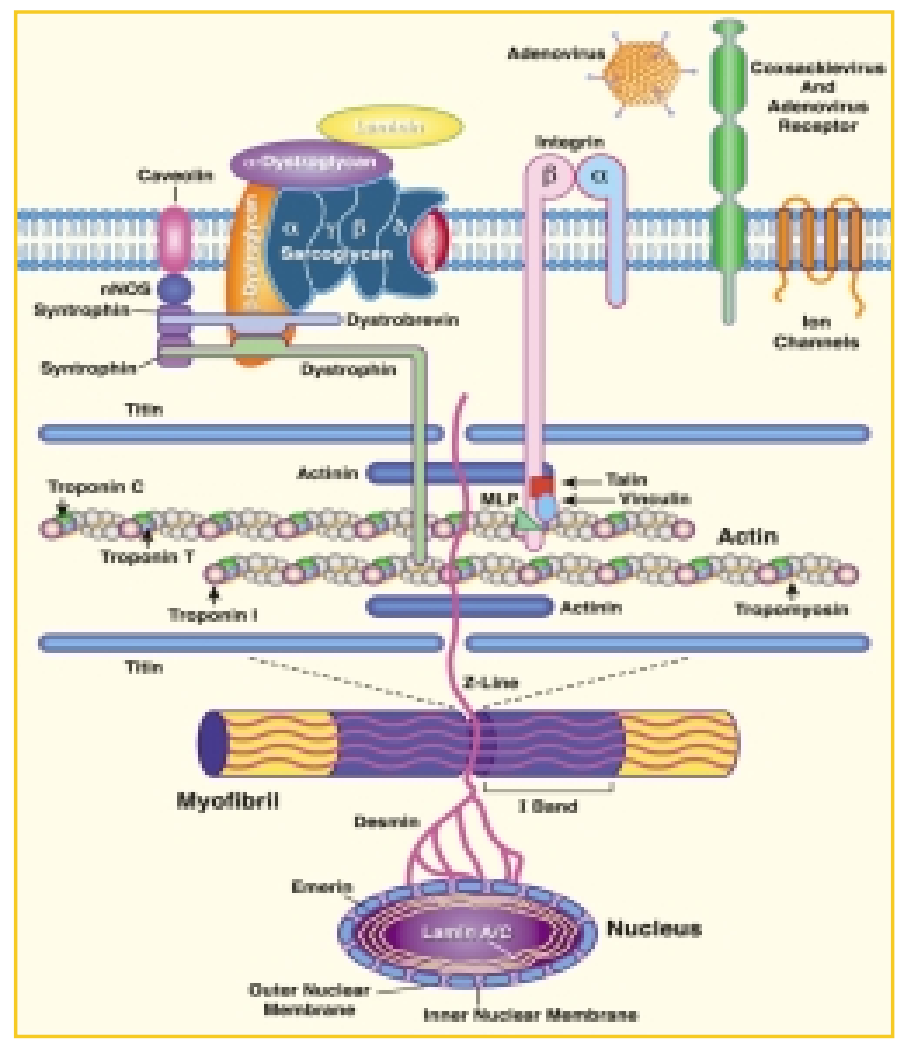

Figure 1

Proteins of the sarcomere, cytoskeleton, sarcolemma, and ECM of muscle, which are connected. M utations in $\delta$-sarcoglycan, dystrophin, actin, desmin, and lamin A/C are known to cause DCM . All of these genes also cause forms of skeletal myopathy when mutated. Mutations in any of the genes illustrated could potentially cause DCM and/or skeletal myopathy, forming the basis of the "final common pathway." Signaling pathways (such as nNos) interacting with these proteins are likely to modify the phenotype. 
angiograms and normal coronary anatomy and histology when explanted (8). H owever, based on themousemode experience and prior data obtained in patients with DCM treated with verapamil, the authors performed the current el egant study, which suggests physiologic improvement resulting from verapamil therapy (1). Whether this is dueto blockade of calcium flux, vasodilation, or protection from ischemia directly is not known; theauthors speculate that the latter mechanism is at play (at least in these mice).

The stage is now set to understand and treat DCM more effectively, as well as improving our global concepts regarding heart muscledisease. First, it is clear that disruption of the sarcomere-sarcolemma linkage results in the clinical phenotype of DCM and that perturbation of this "final common pathway" at various points in the pathway results in the disorder $(12,13)$. Second, patients with DCM are likely to havesubclinical or mild skeletal myopathy, potentially manifested as easy fatigability clinically in the absence of persistent congestive heart failure. With increased survival, it is possible that late-onset skeletal myopathy will become manifest in the future. Third, proteins in this final common pathway, such as thesarcoglycans or other members of the DAPC, likely havemorethan a structural role; signal pathways probably also are in play. In the models pre sented by Cohn et al. (1), it is these cascade pathways that are most likely being influenced by the verapamil, leading to clinical improvement. Finally, it is becoming clear that cardiologists, neurologists, and pharmacologists can positively impact the well-being of patients with this tragic disorder by working together. This will improvethe diagnostic and therapeutic armamentarium necessary to overcome the poor outcome that has been endured by these patients and their families. Caution, however, must be taken in interpreting thefindings described by Cohn et al. (1), as translation from animal models to the human condition is typically fraught with uncertainty.

1. Cohn, R.D., et al. 2001. Prevention of cardiomy opathy in mousemodels lacking thesmooth mus cle sarcoglycan-sarcospan complex. J. Clin. Invest. 107:R1-R7.

2. Coral-Vazquez, R., et al. 1999. Disruption of the sarcoglycan-sarcospan complex in vascular smooth muscle: a novel mechanism for cardiomyopathy and muscular dystrophy. Cell. 98:465-474

3. Towbin, J.A., et al. 1993. X-linked dilated cardiomyopathy (XLCM): molecular genetic evidence of linkage to the Duchenne muscular dystrophy geneat theXp21 locus. Circulation. 87:1854-1865.

4. Olson, T.M., and Keating, M.T. 1996. M apping a cardiomyopathy locus to chromosome 3p22-p25. J. Clin. Invest. 97:528-532.

5. Li, D., et al. 1999. Desmin mutation responsible for idiopathic dilated cardiomyopathy. Circulation. 100:461-464.

6. Fatkin, D., et al. 1999. Missense mutations in the rod domain of the lamin $A / C$ gene as causes of dilated cardiomyopathy and conduction system disease. N. Engl. J. M ed. 341:1715-1724.

7. Brodsky, G.L., et al. 1999. Lamin A/C gene mutation associated with dilated cardiomyopathy with variable skeletal muscle involvement. Circulation. 101:473-476.

8. Tsubata, S., et al. 2000. Mutations in the human delta-sarcoglycan gene in familial and sporadic dilated cardiomyopathy. J. Clin. Invest. 106:655-662.

9. M atsumura, K., et al. 1999. Sarcoglycan complex: a muscular supporter of dystroglycan-dystrophin interplay? Cell. Mol. Biol. 45:751-762.

10. Cox, G.F., and Kunkel, L.M . 1997. Dystrophies and heart disease. Curr. Opin. Cardiol. 12:329-343.

11. M elacini, P., et al. 1998. Heart involvement in muscular dystrophies due to sarcoglycan gene mutations. M uscleNerve. 22:473-479.

12. Towbin, J.A. 1998. Therole of cytoskeletal proteins in cardiomyopathies. Curr. Opin. Cell Biol. 10:131-139.

13. Bowles, N.E., Bowles, K.R., and Towbin, J.A. 2000 The "final common pathway" hypothesis and inherited cardiovascular disease. Herz. 25:168-175. 